\title{
Exploring recognition of affective prosody in patients with remitted depression: how do they differ from healthy participants? Maria Giannakou*1,2, Vasilis P Bozikas ${ }^{3}$, Mary H Kosmidis' ${ }^{1}$, Mihalis Saitis ${ }^{4}$, Aravela Adamopoulou ${ }^{4}$ and George Garyfallos ${ }^{2}$
}

Address: ${ }^{1}$ Neuropsychology Group, School of Psychology, Aristotle University of Thessaloniki, Greece, 2 2nd Department of Psychiatry, Medical School, Aristotle University of Thessaloniki, Greece, ${ }^{3} 1$ st Department of Psychiatry, Medical School, Aristotle University of Thessaloniki, Greece and ${ }^{4}$ Community Mental Health Center, Northwest District of Thessaloniki, Greece

* Corresponding author

from International Society on Brain and Behaviour: 3rd International Congress on Brain and Behaviour

Thessaloniki, Greece. 28 November - 2 December 2007

Published: 17 April 2008

Annals of General Psychiatry 2008, 7(Suppl I):SI6I doi:10.1 186/1744-859X-7-SI-SI6I

This abstract is available from: http://www.annals-general-psychiatry.com/content/7/SI/SI6I

(c) 2008 Giannakou et al.; licensee BioMed Central Ltd.

\section{Background}

Deficits in recognizing emotional states in others have been observed in several psychiatric and neurological disorders. Findings from studies examining emotion processing in depressed patients, however, have been inconsistent. Moreover, the indications of a generalized or a specific emotion recognition impairment have been controversial. Thus, the purpose of the present study was to explore emotion recognition, and, specifically, affective prosody recognition, in a sample of depressed patients in remission.

\section{Materials and methods}

Seventeen patients with remitted depression (RD) and 20 healthy controls (HC), matched on age and education, were assessed with an affective prosody test (APT). In this test, 30 audiorecorded sentences of emotionally neutral content (e.g. "John is studying") were presented with prosodic intonation portraying one of the basic emotions (happiness, sadness, surprise, fear, anger, as well as neutral) with five examples of each emotion.

\section{Results}

Independent samples t-tests revealed no group main effect, either on their overall performance on the APT $[\mathrm{t}(35)=.611, \mathrm{p}=.545]$, or on any particular emotion [happiness: $\mathrm{t}(35)=1.579, \mathrm{p}=.087$; sadness: $\mathrm{t}(35)=-.317$ ， $\mathrm{p}=.753$; surprise: $\mathrm{t}(35)=-1.006, \mathrm{p}=.321$; fear: $\mathrm{t}(35)=1.204$, $\mathrm{p}=.237$; anger: $\mathrm{t}(35)=-1.124, \mathrm{p}=.268$; neutral: $\mathrm{t}(35)=-$
$1.262, \mathrm{p}=.215]$. For the patients, the rank order of the six emotions from the highest to lowest mean score was as follows: neutral intonation, anger, surprise, happiness, sadness, and fear, while for the healthy participants it was as follows: neutral intonation, happiness, fear, surprise, anger and sadness.

\section{Conclusions}

Although the two groups did not differ in their overall performance, patients with remitted depression showed a different pattern of emotion recognition, suggesting subtle deviation in the mode in which they identify emotional states. A larger sample of patients should be assessed, however, in order to strengthen the validity of our conclusion. 